\title{
On the nature of five open cluster candidates projected close to the Galactic centre ${ }^{\star, \star \star}$
}

\author{
A. E. Piatti ${ }^{1}$ and J. J. Clariá ${ }^{2}$ \\ 1 Instituto de Astronomía y Física del Espacio, CC 67, Suc. 28, 1428 Buenos Aires, Argentina \\ 2 Observatorio Astronómico, Universidad Nacional de Córdoba, Laprida 854, 5000 Córdoba, Argentina
}

Received 2 August 2001 / Accepted 26 September 2001

\begin{abstract}
We present CCD BVI observations obtained for 1759 stars in $4^{\prime} \times 4^{\prime}$ regions around the unstudied southern stellar aggregates Ruprecht 103, 124, 129, 146 and 166, which are described as open clusters in several catalogues. Our analysis shows that none of these stellar groups are genuine open clusters since no clear main sequences or other meaningful features can be seen in their colour-magnitude diagrams. Star counts carried out within and outside the cluster candidate fields not only support these results but also show that the studied objects do not appear to be candidate late stages of star cluster dynamical evolution.
\end{abstract}

Key words. open clusters and associations: individual: Ruprecht 103, 124, 129, 146, and 166 - open clusters and associations : general

\section{Introduction}

Galactic open clusters have long been considered unrivalled objects not only to undertake structural and evolutionary studies of our own Galaxy's disk but also to enhance our understanding of stellar formation, structure, and evolution. Galactic open clusters owe this privileged status to the relative ease and precision with which their reddenings, distances, and metallicities can be ascertained (see, e.g., Janes et al. 1988; Twarog et al. 1997). CCD photometric surveys of Galactic open star clusters are currently under way with the aim of obtaining good-quality photometric data not only to enlarge the sample of studied clusters, but also to estimate their fundamental parameters reliably (e.g., Phelps 2001). In some cases, however, the main goal is to explore the star formation history and spatial structure in selected regions (e.g., Moitinho 2001), while in others, the primary aim is to catalogue specific types of stars in selected open clusters (e.g., Kalirai et al. 2001). At the same time, systematic surveys to find new star clusters in selected fields (Dutra \& Bica 2001), as well

Send offprint requests to: A. E. Piatti,

e-mail: andres@iafe.uba.ar

* Based on observations made at the University of Toronto (David Dunlap Observatory) 24-inch telescope, Las Campanas, Chile.

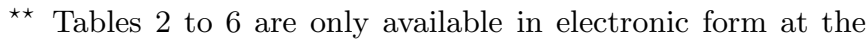
CDS via anonymous ftp to

cdsarc.u-strasbg.fr (130.79.128.5) or via

http://cdsweb.u-strasbg.fr/cgi-bin/qcat?J/A+A/379/453 as open cluster remnants (Bica et al. 2001; Pavani et al. 2001) are also being undertaken.

As is commonly accepted, the presence of an apparent concentration of stars in the sky does not necessarily lead to the inevitable conclusion that such concentration constitutes a physical cluster. Any of the following factors, or a possible combination of them, could account for differences in the star density in some region: the presence of a genuine open cluster, an accidental distribution of stars along the line of sight, or the inhomogeneous distribution of interstellar material. In the last few years, several CCD photometric studies of open cluster candidates included in the Lyngå (1987) catalogue have been carried out with the main purpose of determining whether they are genuine physical systems (see, e.g., Piatti et al. 2000a, and references therein). Even though in some cases, the studied objects have been confirmed as genuine physical systems (see, e.g. Piatti et al. 1998, 2000b), in some others, there exists evidence that the presumed clusters are not real, but rather random fluctuations of stellar density in a given region (e.g., Carraro \& Patat 1995; Piatti \& Clariá 2001). In some cases, the physical nature of the open cluster candidates is still highly debatable. A typical example is NGC 6994, which Bassino et al. (2000) consider a 2-3 Gyr open cluster, but which Carraro (2000) assumes to be simply a random enhancement of four bright stars above the background level rather than an open cluster. Since star clusters are known to evolve dynamically and stellar depletion effects eventually lead to cluster dissolution, it is probable that some unconfirmed clusters are 
in fact cluster remnants or fossil remains (de la Fuente Marcos 1998; Bica et al. 2001).

We have recently started a systematic survey that consists in obtaining colour-magnitude diagrams (CMDs) of numerous, mostly unstudied, poorly populated objects described as star clusters in open cluster catalogues (Alter et al. 1970; Lyngå 1987; Lauberts 1982). The primary purpose of this survey is to determine whether they are real physical systems or not. In this paper we present the results obtained for five open cluster candidates projected close to the Galactic centre, namely: Ruprecht 103 or ESO 130-SC11 (OCL-876, $\alpha_{1950}=12^{\mathrm{h}} 13^{\mathrm{m}} 12^{\mathrm{s}}, \delta_{1950}=$ $-58^{\circ} 08^{\prime} ; l=298.28^{\circ}, b=4.14^{\circ}$ ), Ruprecht 124 (OCL$1005, \alpha_{1950}=17^{\mathrm{h}} 24^{\mathrm{m}} 30^{\mathrm{s}}, \delta_{1950}=-40^{\mathrm{o}} 44^{\prime} ; l=348.07^{\mathrm{o}}$, $b=-3.32^{\circ}$ ), Ruprecht 129 or ESO 455-SC40 (OCL-1037, $\alpha_{1950}=17^{\mathrm{h}} 44^{\mathrm{m}} 06^{\mathrm{s}}, \delta_{1950}=-29^{\circ} 35^{\prime} ; l=359.62^{\mathrm{o}}, b=$ $\left.-0.66^{\circ}\right)$, Ruprecht 146 or ESO 592-SC4 (OCL-39, $\alpha_{1950}=$ $\left.18^{\mathrm{h}} 49^{\mathrm{m}} 36^{\mathrm{s}}, \delta_{1950}=-21^{\mathrm{o}} 11^{\prime} ; l=14.08^{\mathrm{o}}, b=-9.70^{\circ}\right)$ and Ruprecht 166 or ESO 96-SC11 (OCL-899, $\alpha_{1950}=$ $\left.13^{\mathrm{h}} 22^{\mathrm{m}} 42^{\mathrm{s}}, \delta_{1950}=-63^{\mathrm{o}} 09^{\prime} ; l=306.80^{\circ}, b=-0.80^{\circ}\right)$. These five objects were discovered by Ruprecht (1960), who described them as clusters of Trumpler class IV2p, II3p, IV1p, III2p, and III3p, respectively. The ESO identifications are all from Lauberts (1982). Ruprecht 103 is located in Crux, Ruprecht 124 in Scorpius, Ruprecht 166 in Centaurus, and Ruprecht 129 and 146 in Sagittarius, all projected close to the Galactic centre. Only Ruprecht 166 has some information available for it. Moffat \& Vogt (1973) observed three comparatively bright stars in the field of Ruprecht 166 using $U B V$ photolectric photometry and concluded that this is a doubtful cluster in view of the relatively high field star density in the vicinity of the supposed cluster region. In Sect. 2 we present the observations and describe the reduction procedures briefly. We analize in Sect. 3 the possible existence of genuine open clusters within the observed sample, following two different approaches. We discuss the results in Sect. 4, and finally we present the concluding remarks in Sect. 5 .

\section{Observations and reductions}

We carried out CCD observations in the regions of Ruprecht 103, 124, 129, 146 and 166 through the $B$, $V$, and $I$ passbands during four photometric nights in September 1994 and June-July 1995. The direct images were obtained with the 24-inch telescope of the University of Toronto Southern Observatory (Las Campanas Observatory, Chile), equipped with a Photometric $512 \times 512$ Metachrome CCD coated to give improved blue response. The scale on the chip was $0.45^{\prime \prime}$ per pixel, yielding an area covered by a frame of $4^{\prime} \times 4^{\prime}$. Cols. 4 and 5 of Table 1 list the exposure sequences and the typical seeing for each object and filter, while Fig. 1 shows the schematic finding-charts built with all the measured stars in the $V$-band. Observations of 12-17 stars in the Selected Area standard fields (Landolt 1992) covering a wide range in colour $(-0.12 \leq V-I \leq 2.05)$ were also taken nightly. Furthermore, a series of 10 bias exposures

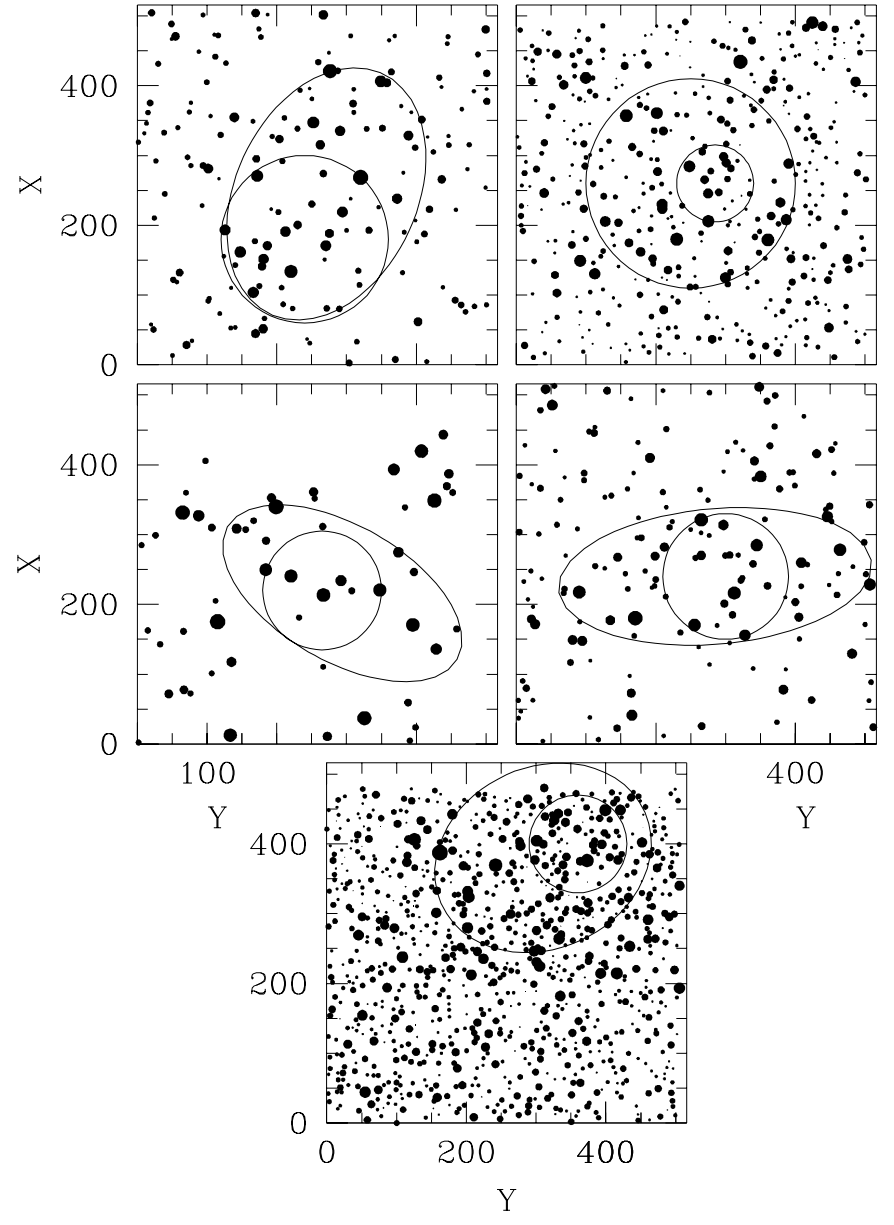

Fig. 1. Schematic finding charts for the fields of Ruprecht 103 (top-left), Ruprecht 124 (top-right), Ruprecht 129 (middleleft), Ruprecht 146 (middle-right), and Ruprecht 166 (bottom). Two circular and elliptical extractions are also shown. North is up and East is to the left. The sizes of the filled circles are proportional to the brightness of the stars.

and 10 flat-field frames of both the twilight sky and dome were taken for CCD calibration purposes. The airmass values for the observed fields were always smaller than 1.35, just lying within the airmass range of the standard stars.

The observations were reduced at the Observatorio Astronómico de la Universidad Nacional de Córdoba (Argentina), using the $\operatorname{IRAF}^{1}$ routines. The $b, v$ and $i$ instrumental magnitudes were obtained following the reduction procedure described by Piatti et al. (1999) and transformed into the standard system using the following relations:

$$
\begin{aligned}
& b_{j, n}=b_{1}+V+(B-V)+b_{2}(B-V)+b_{3} X_{j, n} \\
& v_{k, n}=v_{1}+V+v_{2}(B-V)+v_{3} X_{k, n} \\
& i_{l, n}=i_{1}+V-(V-I)+i_{2}(V-I)+i_{3} X_{l, n}
\end{aligned}
$$

${ }^{1}$ IRAF is distributed by the National Optical Astronomy Observatories, which is operated by the Association of Universities for Research in Astronomy, Inc., under contract with the National Science Foundation 
Table 1. Journal of observation.

\begin{tabular}{lcccc}
\hline Object & $\begin{array}{c}\text { Date } \\
\text { (UT) }\end{array}$ & Filter & Exposures & $\begin{array}{c}\text { seeing } \\
\left({ }^{\prime \prime}\right)\end{array}$ \\
\hline Ruprecht 103 & 1995 June 28 & $B$ & $1 \times 60 \mathrm{~s}, 2 \times 300 \mathrm{~s}$ & 1.3 \\
& & $V$ & $1 \times 60 \mathrm{~s}, 2 \times 200 \mathrm{~s}$ & 1.3 \\
Ruprecht 124 & \multirow{2}{*}{ 1995 July 2 } & $B$ & $1 \times 30 \mathrm{~s}, 2 \times 60 \mathrm{~s}$ & 1.3 \\
& & $V$ & $2 \times 900 \mathrm{~s}$ & 1.5 \\
& & $I$ & $1 \times 60 \mathrm{~s}, 2 \times 300 \mathrm{~s}$ & 1.5 \\
Ruprecht 129 & \multirow{2}{*}{ 1994 September 3 } & $B$ & $1 \times 80 \mathrm{~s}, 1 \times 400 \mathrm{~s}, 1 \times 500 \mathrm{~s}$ & 1.4 \\
& & $V$ & $1 \times 80 \mathrm{~s}, 1 \times 250 \mathrm{~s}$ & 1.4 \\
& & $I$ & $1 \times 5 \mathrm{~s}, 2 \times 30 \mathrm{~s}$ & 1.4 \\
Ruprecht 146 & 1994 September 3 & $B$ & $1 \times 80 \mathrm{~s}, 1 \times 600 \mathrm{~s}$ & 1.4 \\
& & $V$ & $1 \times 100 \mathrm{~s}, 2 \times 250 \mathrm{~s}$ & 1.4 \\
Ruprecht 166 & 1995 June 30 & $B$ & $1 \times 20 \mathrm{~s}, 2 \times 50 \mathrm{~s}$ & 1.4 \\
& & $V$ & $1 \times 60 \mathrm{~s}, 2 \times 600 \mathrm{~s}, 2 \times 300 \mathrm{~s}$ & 1.6 \\
& & $I$ & $1 \times 30 \mathrm{~s}, 2 \times 150 \mathrm{~s}$ & 1.6 \\
\hline
\end{tabular}

Table 2. Magnitudes and colours of stars in the field of Ruprecht 103.

\begin{tabular}{cccccccccc}
\hline ID & $X_{\text {pixels }}$ & $Y_{\text {pixels }}$ & $V$ & $\sigma(V)$ & $B-V$ & $\sigma(B-V)$ & $V-I$ & $\sigma(V-I)$ & $n$ \\
\hline 1 & 331.916 & 10.242 & 18.383 & 0.051 & 1.045 & 0.144 & 1.308 & 0.064 & 2 \\
2 & 361.558 & 15.393 & 17.552 & 0.032 & 1.314 & 0.089 & 1.398 & 0.034 & 2 \\
3 & 504.436 & 20.719 & 16.418 & 0.016 & 0.962 & 0.031 & 1.017 & 0.022 & 1 \\
4 & 50.748 & 23.507 & 16.929 & 0.020 & 1.591 & 0.057 & 1.625 & 0.023 & 2 \\
5 & 210.538 & 24.632 & 17.651 & 0.032 & 0.933 & 0.072 & 1.137 & 0.037 & 2 \\
. &. &. &. &. &. &. &. &. &. \\
. &. &. &. &. &. &. &. &. &. \\
. &. &. &. &. &. &. &. &. &. \\
\hline
\end{tabular}

NOTE: Tables 2 to 6 are available in their entirety in electronic form at the CDS via anonymous ftp to cdsarc.u-strasbg.fr (130.79.128.5) or via http://cdsweb.u-strasbg.fr/cgi-bin/qcat?/A+A/379/453. A portion of Table 2 is shown here for guidance regarding its form and content.

where $V,(B-V)$ and $(V-I)$ are the standard magnitude and colours and $X$ the corresponding airmass for the $j$, $k, l$ th measured standard star. Equations (1) to (3) were solved for all coefficients simultaneously for each night $n$ with the PHOTCAL package in IRAF. The mean rms errors range between $0.005 \mathrm{mag}$ and $0.020 \mathrm{mag}$ in all the passbands, which indicate that the nights were all of good photometric quality. Figure 2 shows the trend of the photometric magnitude and colour errors with $V$ provided by DAOPHOT for the richest observed field (see Sect. 3). For each selected field, we generated a master table containing a running star number, the $X$ and $Y$ coordinates, the $V$ magnitude, the $B-V$ and $V-I$ colours, the observational errors provided by the IRAF INVERTFIT task $\sigma(V), \sigma(B-V)$ and $\sigma(V-I)$, and the number of observations $n$. These tables were built by combining all the independent measurements using the stand-alone DAOMATCH and DAOMASTER programmes kindly provided by Peter Stetson. Tables 2 to 6 provide this information and are available in electronic form at the CDS. A portion of Table 2 is shown here for guidance regarding its form and content.
A comparison of our CCD photometry with Moffat \& Vogt (1973) for 3 stars in common in the field of Ruprecht 166 yields $\Delta\left(V_{\mathrm{MV} 73}-V_{\mathrm{CCD}}\right)=0.022 \pm 0.045$ and $\Delta((B-$ $\left.V)_{\mathrm{MV} 73}-(B-V)_{\mathrm{CCD}}\right)=0.010 \pm 0.031$.

\section{Data analysis}

As shown in Fig. 1, fields differ from each other in terms of the noticeable contrast in their stellar densities: Ruprecht 129 and Ruprecht 166 are the fields with the smallest and largest numbers of stars, respectively, the latter having more than 17 times the number of stars measured for the former. This result corroborates the fact that sky regions close to the direction towards the Galactic centre present strong variations in the stellar density at a fixed limiting magnitude, even though they are separated by few degrees. Taking into account these stellar density variations, an open cluster candidate might be identified by the concentration of a handful of bright stars which stand out from a slightly fainter surrounding field, or by a visible increase in the stellar population in a sky region, or by both criteria combined. Occasionally, apparent 


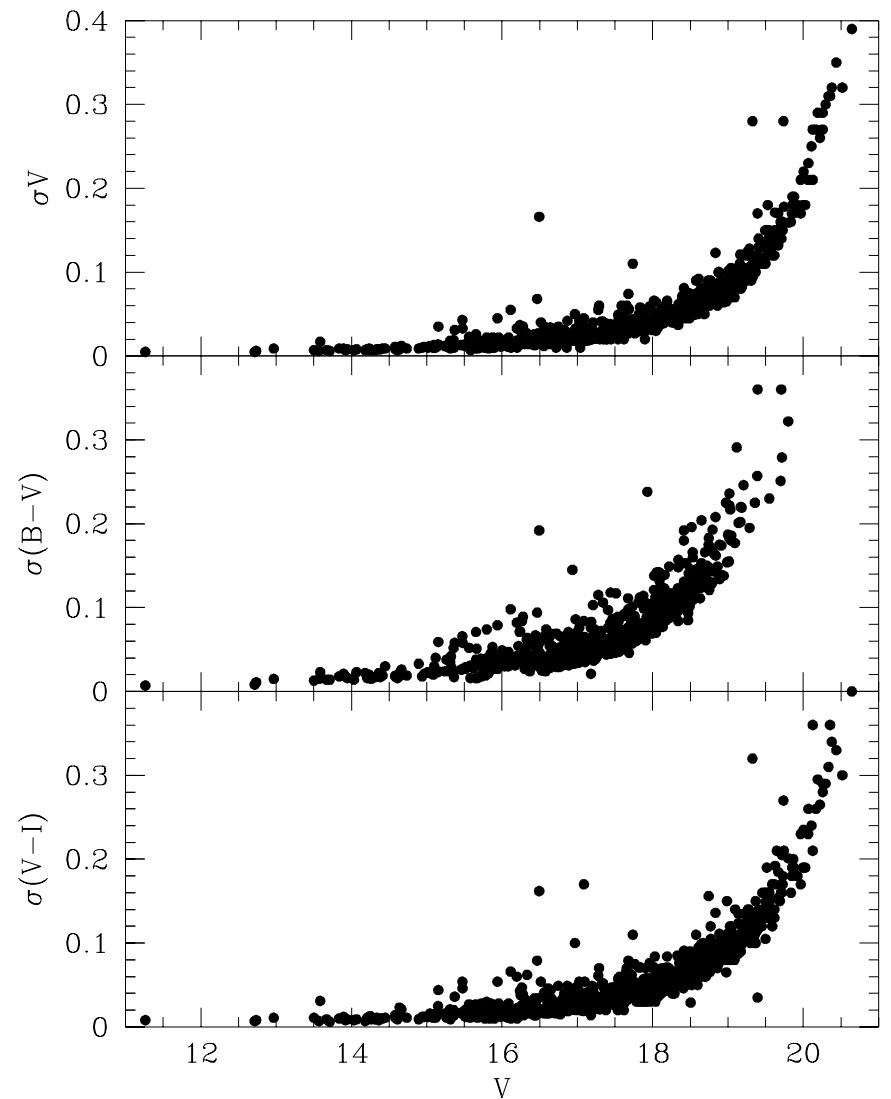

Fig. 2. Magnitude and colour photometric errors provided by DAOPHOT as a function of $V$ for the richest cluster of the sample (Ruprecht 166). They are typical in our sample.

concentrations of bright stars located approximately along the same direction, or variations in the number of stars caused by the presence of interstellar clouds can lead to identification of unreal open clusters.

We analysed the possible existence of genuine open clusters within the object sample following two different approaches. On the one hand, we investigated the distribution of stars in the $(V, B-V)$ and $(V, V-I)$ CMDs and, on the other hand, we compared the number of stars counted within and outside the cluster candidate fields. From the complementary analysis of both approaches we will achieve a more robust confirmation of the physical reality of these objects.

\subsection{Colour-magnitude diagrams}

Since the size of our field of view $\left(F O V \approx 4^{\prime} \times 4^{\prime}\right)$ was considered rather small to outline the boundaries of all the cluster candidates, we used Digitized Sky Survey (DSS) images of $15^{\prime}$ on a side to have a wider view of the selected fields. The fact that the selected objects cannot be embraced by our CCD images suggests that cluster candidates can be more extended than the area covered by the detector and/or that the observed fields do not differ clearly, in terms of stellar density, from their surrounding fields. A wider FOV thus provides a better framework for

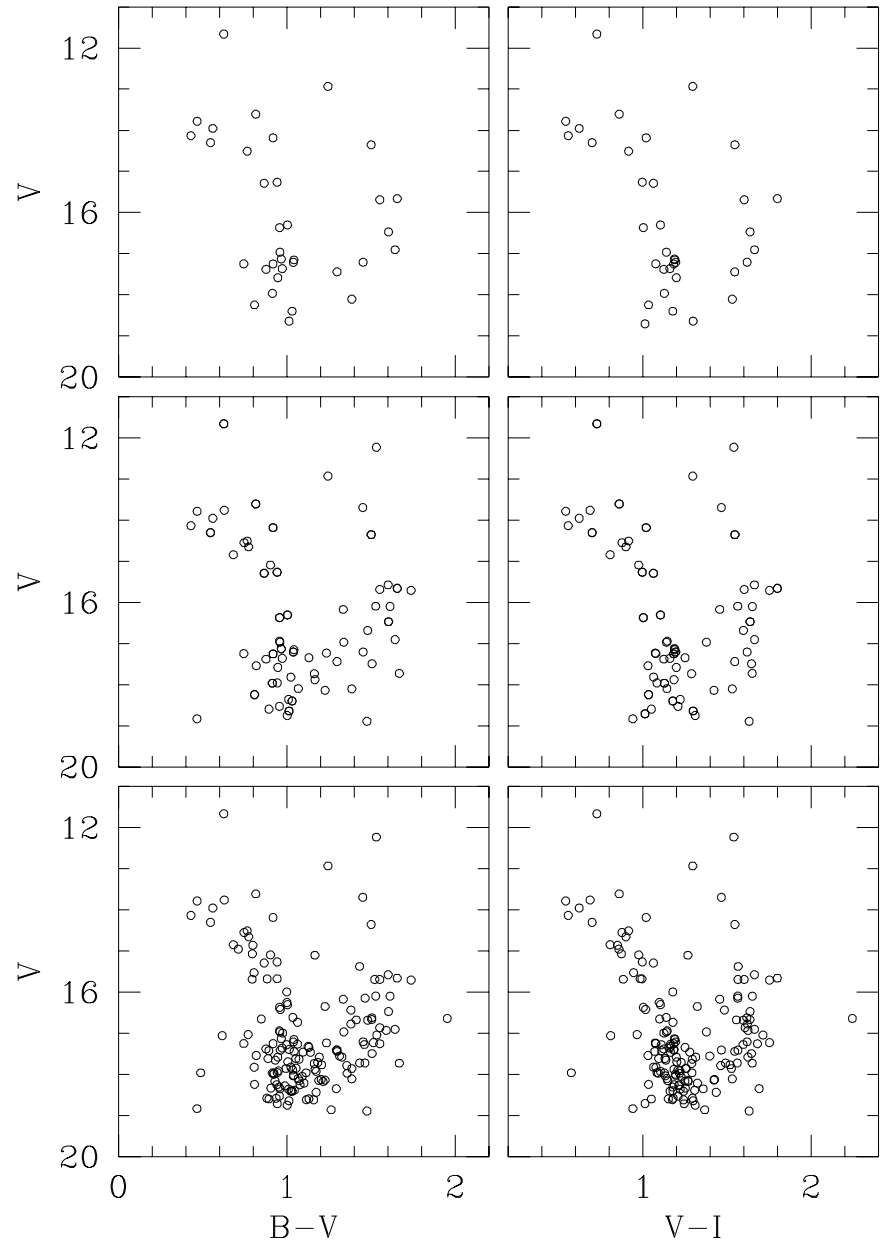

Fig. 3. Colour-magnitude diagrams of stars in the field of Ruprecht 103. Stars within the circular (top) and elliptical (middle) extractions, as well as all measured stars (bottom) are shown.

distinguishing the whole group of stars catalogued as a cluster candidate.

Bearing in mind that open clusters commonly have stars distributed following a non-spherical symmetry, for each object we traced a small circle around what appeared to be its central region, and an ellipse containing the circle to consider the whole extension of the cluster candidate. This ellipse resulted in a compromise between maximizing the object area and minimizing the field star contamination. We overplotted the chosen circles and ellipses on the schematic finding charts of Fig. 1. Cluster candidates are mostly centered on and entirely contained in the observed fields.

We then built $(V, B-V)$ and $(V, V-I)$ CMDs for the circular and elliptical extractions as well as for all the measured stars. These CMDs are shown in Figs. 3 to 7 for Ruprecht 103, 124, 129, 146 and 166, respectively. The upper panels in the figures correspond to the small circular extractions, whereas those in the middle and at the bottom of the figures are from the elliptical and total fields, respectively. If one of the observed candidates was an open cluster, we would expect to be able to define its 


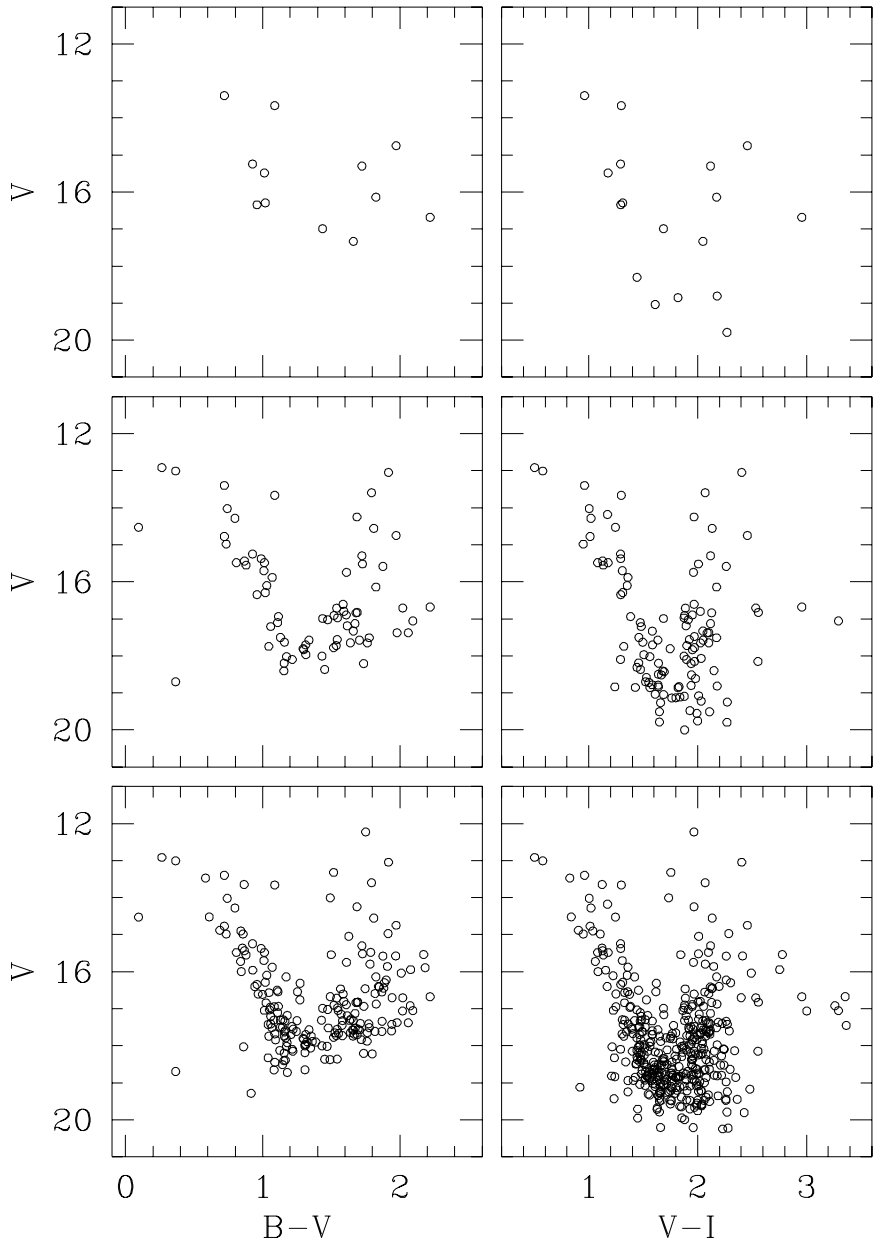

Fig. 4. Colour-magnitude diagrams of stars in the field of Ruprecht 124. Stars within the circular (top) and elliptical (middle) extractions, as well as all measured stars (bottom) are shown.

fiducial Main Sequence (MS) from the circular extraction, and recognize all the cluster features and the unavoidable field star contamination from the elliptical one. Note that the Zero Age Main Sequence (ZAMS) does have a different curvature than the field MS, which follows a lower envelope and depends on the interstellar extinction parameters, namely $R=A_{\mathrm{v}} / E(B-V)$ (Burki \& Maeder 1973). However, none of the extracted CMDs seems to reveal the presence of an open cluster MS, except possibly in the case of Ruprecht 166 (see discussion below). The star sequences seen in the distinct CMDs, specially for Ruprecht 124 and Ruprecht 129, appear to be formed from the superimposition of field stars of several spectral types affected by different amounts of interstellar absorption and/or located at different distances from the Sun.

The blue star sequence in the CMDs of Ruprecht 103 extends down to $V \sim 16 \mathrm{mag}$, where it undergoes a remarkable break and apparently becomes a vertical sequence at fainter magnitudes. This break is not typical of a cluster MS, thus favouring the conclusion that Ruprecht 103 is not an open cluster. Moreover, even

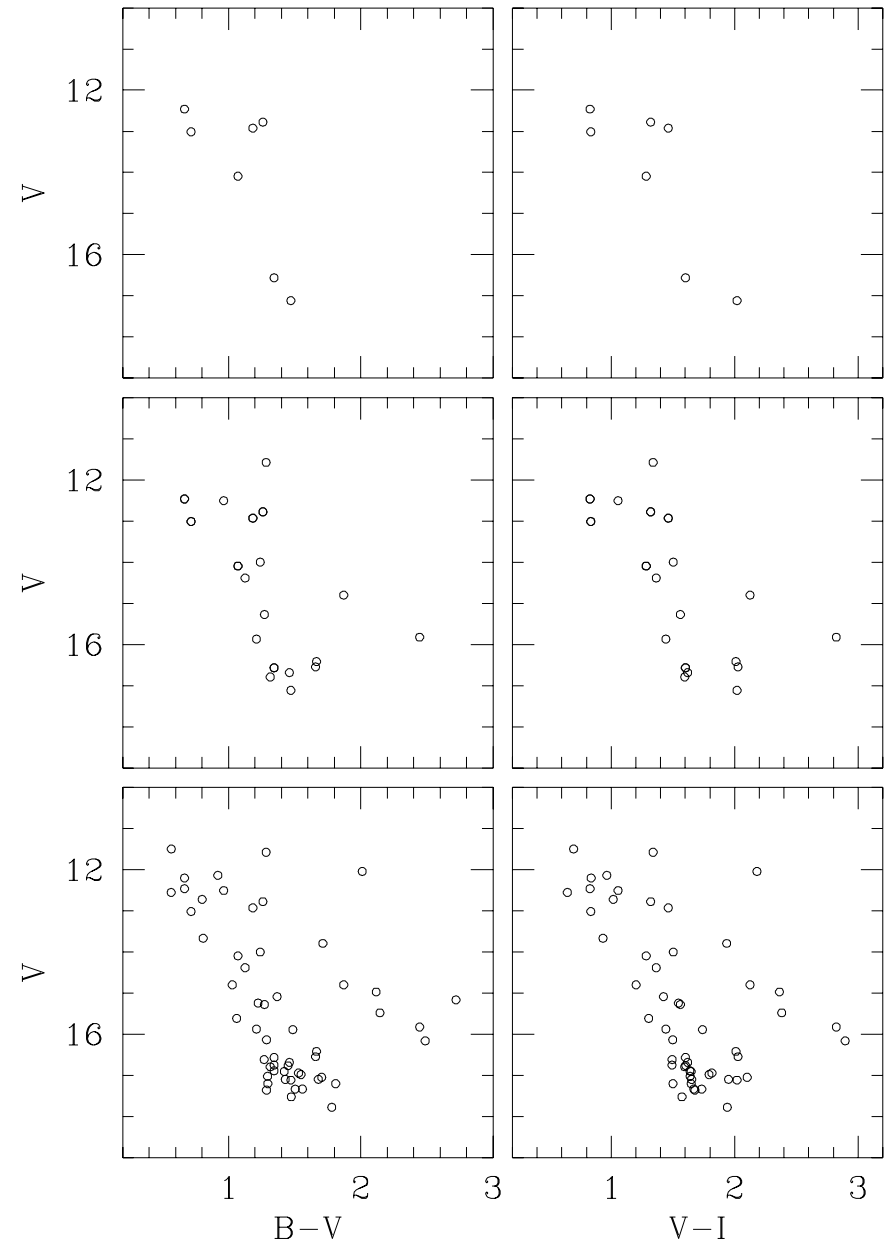

Fig. 5. Colour-magnitude diagrams of stars in the field of Ruprecht 129. Stars within the circular (top) and elliptical (middle) extractions, as well as all measured stars (bottom) are shown.

considering the tilted sequence defined only by the bright stars, we were not able to find a young cluster with a main sequence as inclined as the one we observed. Notice that, under the same assumption, the object could not be of intermediate age or older because of the lack of fainter stars. On the other hand, the bigger the area of the extraction is, the more populated the same parts of the CMDs prove to be, which is an indicator that we are dealing with a uniform field star distribution. Similar characteristics can be recognized with fewer and more dispersed field MS stars in the CMDs of Ruprecht 146, which cannot be confirmed as an open cluster either. The CMDs of Ruprecht 124 and Ruprecht 129 show star sequences that cover a magnitude range of more than 5 mags and have inclinations similar to those of Ruprecht 103 and Ruprecht 146. These results, in addition to the increase in the number of stars outside of the tilted sequence with the extracted area in the field of Ruprecht 124, and the noticeable broadness of the star sequence in the CMDs of Ruprecht 129, are strong evidence against the physical reality of these objects as open clusters. 


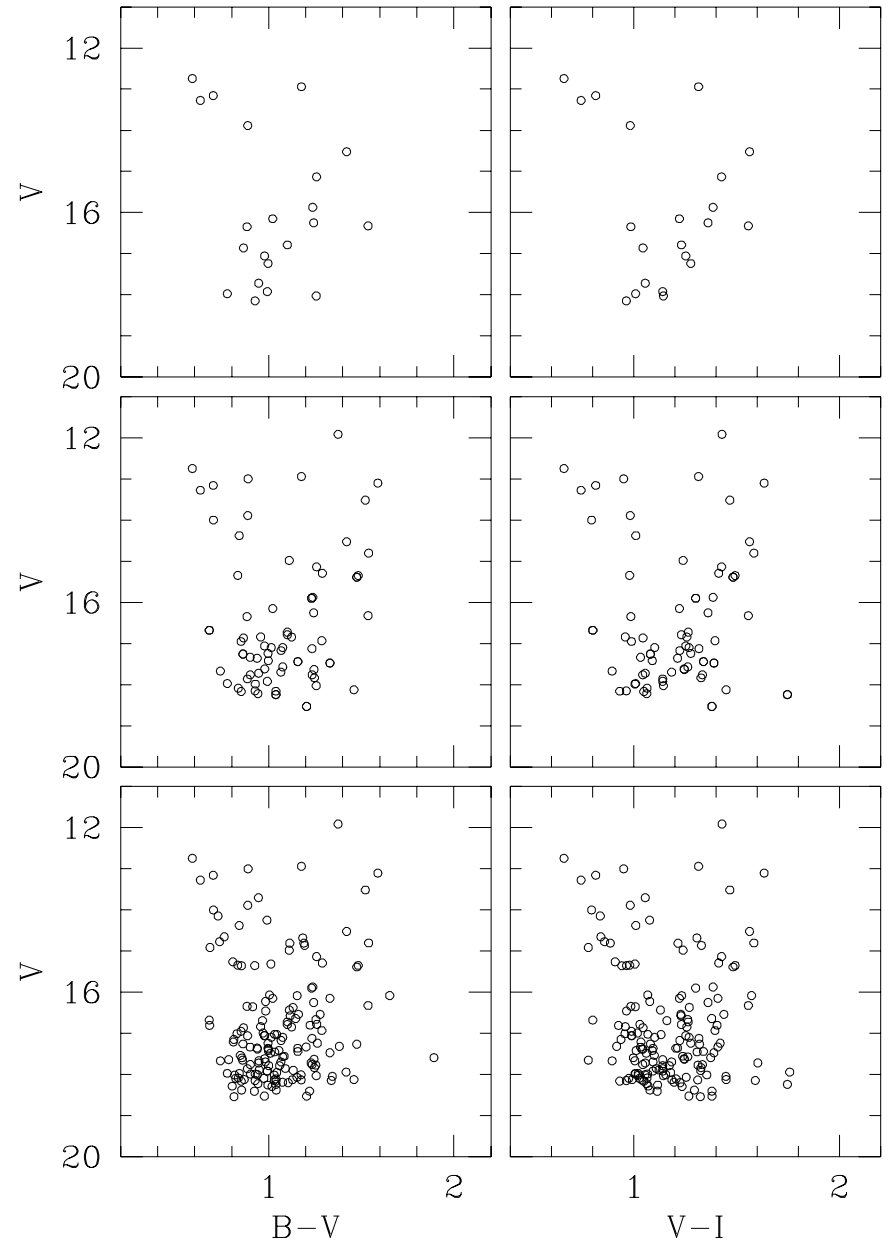

Fig. 6. Colour-magnitude diagrams of stars in the field of Ruprecht 146. Stars within the circular (top) and elliptical (middle) extractions, as well as all measured stars (bottom) are shown.

Finally, the case of Ruprecht 166 deserves particular attention. Its CMDs for the innermost regions appear to show an evolved upper MS, typical of intermediate age open clusters, which extends towards fainter magnitudes with a very important dispersion. The two brightest stars located to the upper-left part of the CMDs from the apparent MS should be considered field stars, if we wanted to keep the category of an intermediate age open cluster for the object. However, the $(V, B-V)$ CMD for the elliptical extraction reveals that the apparent cluster MS is composed by a relatively tight star sequence, extended from $V \sim 13$ mag down to $V \sim 17$ mag, and by a populous clump of stars centered at $(V, B-V) \sim(18,1.1)$. Both sequence and clump of stars have their counterparts in the $(V, V-I)$ CMDs masked like a long MS. Unexpectedly, however, there is not only an abrupt change in the luminosity function along this apparent star sequence (lower panel of Fig. 7), but also an offset for faint stars towards redder colours, which suggest a different origin for these stars. Additionally, it is not possible to fit a ZAMS to the stars in the sequence and in the clump, simultaneously.
Table 7. Stellar densities derived for the selected fields

\begin{tabular}{lcc}
\hline Name & $\begin{array}{c}N_{\text {obj }} \\
\left(\text { stars } / \operatorname{arcmin}^{2}\right)\end{array}$ & $\begin{array}{c}N_{\text {field }} \\
\left(\text { stars } / \operatorname{arcmin}^{2}\right)\end{array}$ \\
\hline Ruprecht 103 & 11 & $9 \pm 1$ \\
Ruprecht 124 & 13 & $13 \pm 1$ \\
Ruprecht 129 & 6 & $7 \pm 1$ \\
Ruprecht 146 & 6 & $5 \pm 1$ \\
Ruprecht 166 & 26 & $22 \pm 2$ \\
\hline
\end{tabular}

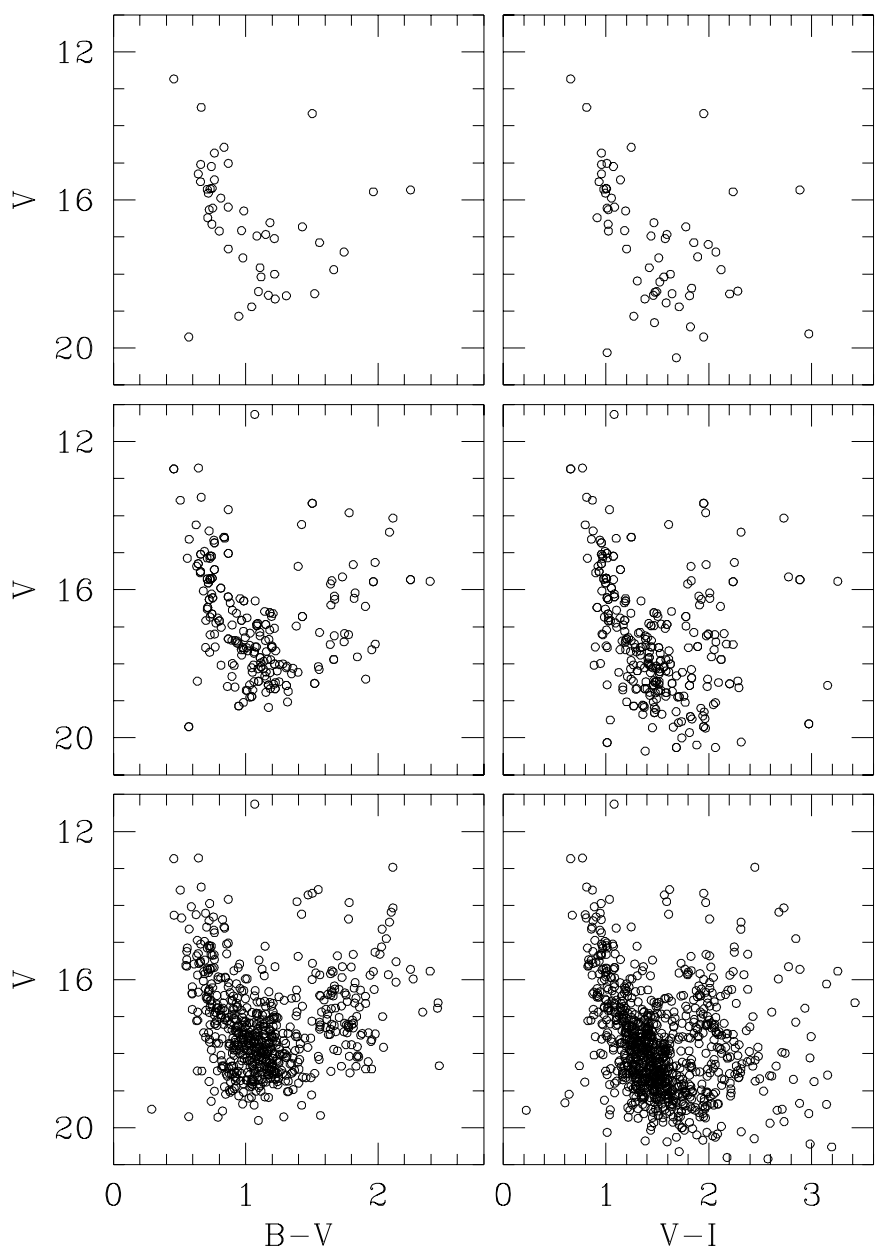

Fig. 7. Colour-magnitude diagrams of stars in the field of Ruprecht 166. Stars within the circular (top) and elliptical (middle) extractions, as well as all measured stars (bottom) are shown.

\subsection{Star counts}

We performed star counts on the DSS images with the aim of looking for some excess of stars in the observed fields that could favour the status of the catalogued objects as open clusters. Firstly, we used the FIND task within the stand-alone version of the DAOPHOT II package (Stetson 1994) to identify as many stars as possible. We were particularly cautious in the assessment of the appropriate threshold to include in the coordinate list only those 


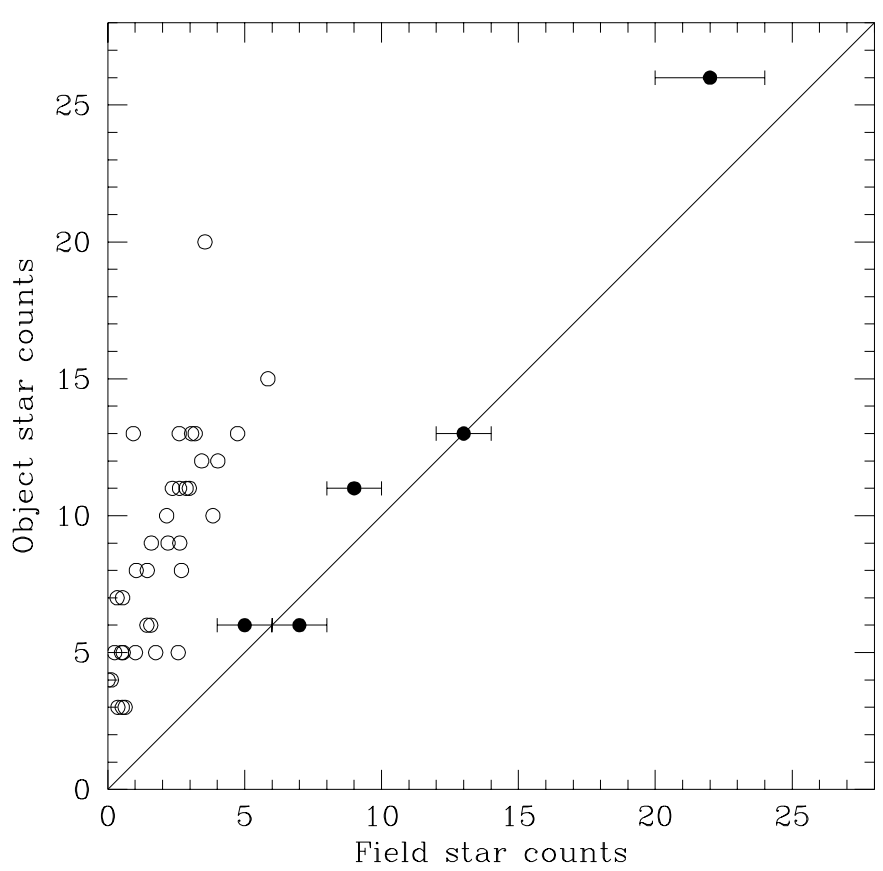

Fig. 8. Relationship between the number of stars per square arcmin estimated for the object and its surrounding field. Open circles represent the values obtained by Bica et al. (2001) whereas filled circles correspond to results of Table 7 . The solid line corresponds to the same number of stars for object and field, respectively.

objects having stellar profiles. In addition, we checked the identified stars in the DSS images. Secondly, we counted the number of stars within circles of radius $\theta_{\mathrm{obj}}$, where $\theta_{\mathrm{obj}}$ represents the apparent major radius of each object. This conservative criterion of using circular regions for counting field stars that encompass the object ellipse is an attempt to compensate for the uncertainties in the defined object boundaries. We counted field stars within 100 circles selected randomly and spread throughout each DSS image, discarding those circular regions superimposed on a circle of radius $\theta_{\text {obj }}$ centered on the object. The resulting mean values of the number of field stars $\left(N_{\text {field }}\right)$, together with their corresponding standard deviations $\left(\sigma_{N_{\text {field }}}\right)$ and the number of stars counted for the objects (same area as for the respective field) are listed in Table 7 . As can be seen, object and field densities turned out to be indistinguishable within one or two $\sigma_{N_{\text {field }}}$.

\section{Discussion}

We compared our results from the stellar counts with those obtained by Bica et al. (2001) for 34 physical systems, catalogued by the authors as possible star clusters in the process of disruption or their fossil remains. Their results show that these possible open cluster remnants, represented by open circles in Fig. 8, are clearly distributed above the line corresponding to regions with uniform stellar densities. On the contrary, our Ruprecht fields are placed very close to this line, Ruprecht 166 having the highest stellar density and the biggest difference between the number of stars counted for the object and for the surrounding field. This result not only is in excellent agreement with the conclusions inferred from the CMD analysis (see Sect. 3.1), in the sense that the studied catalogued objects are not genuine open clusters, but also shows that they do not appear to be candidates of late stages in the star cluster dynamical evolution. According to de la Fuente Marcos (1998), who studied the likely observational properties of open cluster remnants from numerical simulations, a loose cluster should be about ten times denser than the surrounding fields in order to be detected. Hence, it would appear that Ruprecht's classification of the object sample could have been motivated by the apparent concentration of some bright field stars rather than from a visible excess of stars.

With the purpose of investigating the origin of stars in the CMDs of the observed fields, we used the statistical results obtained by $\mathrm{Ng}$ et al. (1996) from the decomposition of the disk populations in CMDs of fields towards the Baade's Window. Based on their findings, we identified different stellar disk components in the observed CMDs, namely: the very young disk MS (age $\sim 0.1-2.0 \mathrm{Gyr}$, $Z \approx 0.018-0.022$ ), the old disk MS (age $~ 7.0-10.0 \mathrm{Gyr}$, $Z \approx 0.003-0.008)$, the intermediate age and old disk Horizontal Branches (HBs) and the intermediate age and old disk Red Giants (RGs). The age and metallicity for the intermediate age disk entered by $\mathrm{Ng}$ et al. (1996) are 4.57.0 Gyr and 0.008-0.015 dex, respectively. Thus, in the CMDs of Ruprecht 103 the tilted sequence is composed by very young MS stars, while the fainter vertical sequence is formed by intermediate age and old RG stars. HB stars belonging to the intermediate age and old disks form the red$\operatorname{der}(V-I \sim 1.6)$ and slightly brighter vertical sequence in the CMDs of Ruprecht 103. The same stellar components can be recognized in the CMDs of Ruprecht 146, where faint MS and HB stars have a smaller colour difference. HB stars are also present in the CMDs of Ruprecht 124 and Ruprecht 166, forming a broad vertical sequence centered at $V-I \sim 2.0$. The CMDs of Ruprecht 124 show a more extended MS composed by very young and young stars and by a remarkable old disk star population at its fainter portion $(V \sim 18-20)$. MS stars dominate the CMDs of Ruprecht 129. Finally, the rich star concentration of stars fainter than $V \sim 17 \mathrm{mag}$ and at $V-I \sim 1.0-2.0$ in the CMDs of Ruprecht 166 mainly corresponds to the contribution of old RG stars.

\section{Conclusions}

We report on CCD BVI Johnson-Cousins photometry of stars in the fields of the southern stellar aggregates Ruprecht 103, 124, 129, 146 and 166, all of them included in open cluster catalogues (Alter et al. 1970; Lyngå 1987; Lauberts 1982). None of the observed $(V, B-V)$ and $(V, V-I)$ CMDs reveal the presence of an open 
cluster MS, except perhaps in the case of Ruprecht 166. The star sequences seen in the distinct CMDs, specially for Ruprecht 124 and Ruprecht 129, are apparently formed by the superimposition of MS field stars affected by varying amounts of interstellar absorption and/or placed at different distances from the Sun. The CMDs of Ruprecht 166 present a pronounced variation in the luminosity function along the apparent MS, a fact from which we can infer the notion that these stars had a different origin. We discuss the possible physical nature of the five studied objects by checking whether they present a significant number density contrast with respect to the Galactic field. Star counts performed within and outside the cluster candidate fields not only support the CMDs results but also suggest that the studied objects do not appear to be open cluster remnants.

Several other stellar aggregates included in open cluster catalogues have previously been disproved as genuine physical systems (see, e.g., Carraro \& Patat 1995; Carraro 2000; Piatti \& Clariá 2001). Thus, the present work implies the potential likelihood of other wrongly classified open clusters.

Acknowledgements. We have used DSS images for the analysis. The DSS was produced at the Space Telescope Science Institute under U.S. Government grant NAG W-2166. The images of these surveys are based on photographic data obtained using the Oschin Schmidt Telescope on Palomar Mountain and the UK Schmidt Telescope. We wish to thank the Director, staff and technicians of Las Campanas Observatory for the allocation of observing time and for their kind assistance and hospitality during the observing runs. We gratefully acknowledge financial support from the Argentinian institutions CONICET, SECYT (Universidad Nacional de Córdoba), and Agencia Córdoba Ciencia.

\section{References}

Alter, G., Ruprecht, J., \& Vanisek, J. 1970, Catalogue of Star Clusters and Associations, ed. G. Alter, B. Balázs, \& J. Ruprecht (Akademiai Kiado, Budapest)

Bassino, L. P., Waldhausen, S., \& Martínez, R. E. 2000, A\&A, 355,138

Bica, E., Santiago, B. X., Dutra, C. M., et al. 2001, A\&A, 827, 833

Burki, G., \& Maeder, A. 1973, A\&A, 25, 71

Carraro, G. 2000, A\&A, 357, 145

Carraro, G., \& Patat, F. 1995, MNRAS, 276, 563

de la Fuente Marcos, R. 1998, A\&A, 333, L27

Dutra, C. M., \& Bica, E. 2001, A\&A, 359, L9

Janes, K. A., Tilley, C., \& Lyngå G. 1988, AJ, 96, 771

Kalirai, J. S., Richer, H. B., Fahlman, G. G., et al. 2001, AJ, 122,257

Landolt, A. U. 1992, AJ, 104, 340

Lauberts, A. 1982, The ESO/Uppsala Survey of the ESO (B) Atlas, European Southern Observatory, Garching

Lyngå, G. 1987, Catalogue of Open Cluster Data, Strasbourg: Centre de Données Stellaires

Moffat, A. F. J., \& Vogt, N. 1973, A\&AS, 10, 135

Moitinho, A. 2001, A\&A 370, 436

Ng, Y.K., Bertelli, G., Chiosi, C., \& Bressan, A. 1996, A\&A, 310, 771

Pavani, D. B., Bica, E., Dutra, C. M., et al. 2001, A\&A, 374, 554

Phelps, R. L. 2001, in Extragalactic Star Clusters, ed. D. Geisler, E. Grevel, \& D. Minniti, IAU Symp., 207, in press

Piatti, A. E., Clariá, J. J. 2001, A\&A, 370, 931

Piatti, A. E., Clariá, J. J., Bica, Geisler, D., \& Minniti, D. 1998, AJ, 116, 801

Piatti, A. E., Clariá, J. J., \& Bica, E. 1999, MNRAS, 303, 65

Piatti, A. E., Bica, E., \& Clariá, J. J. 2000a, A\&A, 362, 959

Piatti, A. E., Clariá, J. J., \& Bica, E. 2000b, A\&A, 360, 529

Ruprecht, J. 1960, BAC, 1 (2), 2

Stetson, P. B. 1994, PASP, 106, 250

Twarog, B. A., Ashman, K. M., \& Anthony-Twarog, B. J. 1997, AJ, 114, 2556 